\title{
ZUR DISKURSIVEN UND MODALEN FUNKTION DER PARTIKELN ABER, AUCH, DOCH UND JA IN INSTRUKTIONSDIALOGEN
}

\section{Einleitung:}

Im folgenden geht es um zwei Funktionsbereiche der Partikeln aber, auch, doch und ja, die gemeinhin der Pragmatik zugerechnet werden. Zum einen werden wir die diskursive Funktion, also die verschiedenen Aufgaben dieser Partikeln bei der Organisation und Strukturierung des Gesprächsablaufs, untersuchen. Die Strukturierungseigenschaften der Diskurspartikeln betreffen die Argumentsteuerung und den Dialogablauf, ebenso wie die Regulation der Beziehungsebene zwischen den Kommunikationspartnern und die Organisation der Rede. Zum anderen geht es um die Funktion dieser Lexeme als Modalpartikeln (auch Abtönungspartikeln genannt, z.B. Burkhart 1994). Diese Funktion definieren wir als Indizierung des pragmatischen Prätextes, d.h. als Verweis auf eine vom Sprecher als relevant vorausgesetzte Proposition, an die durch die partikelhaltige Äußerung angeknüpft und die gleichzeitig modifiziert wird.

Das Ziel dieses Aufsatzes ist ein zweifaches: Erstens soll gezeigt werden, daß den verschiedenen Funktionen der Partikeln ein abstraktes semantisches Schema zugrunde liegt, das wir als relationale, genauer als verweisende bzw. indexikalische, Struktur spezifizieren werden. Diese relationale Basisstruktur qualifiziert die interaktiven und modalen Funktionen der Partikeln als grammatische Funktionen. Zweitens werden wir zeigen, daß die semantischen Oppositionen der vier Partikellexeme keineswegs diffus oder gar linguistisch nicht beschreibbar sind, sondern mit wenigen Merkmalen notiert werden können. Vorwegzuschicken ist hier, daß mit dieser Analyse nur die konstantbleibende Kernbedeutung der Partikeln erfaßt werden soll, kontextuelle Nuancierungen, z.B. unter Kontrast oder Emphase, oder Partikelverkettungen werden nicht beschrieben. Sie sollten sich jedoch aus der Kernbedeutung ableiten lassen. Dieser Zielsetzung liegen die folgenden vier Thesen zugrunde:

1.) Die genannten "pragmatischen" Funktionen der Partikeln sind Bestandteil der Grammatik, wobei Grammatik hier zu verstehen ist als diejenigen Regeln und Mechanismen, die dazu dienen, das, was wir sagen wollen, also den darzustellenden kognitiven Inhalt, im linearen Kontinuum der Zeichenproduktion anzuordnen. Diese Anordnung betrifft nicht nur die Enkodierung des 
propositionalen Gehalts, sondern auch seine Verankerung im Textkontinuum und seine Verortung in Bezug auf die kommunikative Situation.

2.) Die Partikeln weisen ihr Funktionsspektrum nicht zufällig auf. Vielmehr lassen sich alle Funktionen auf eine gemeinsame relationale Basissemantik zurückführen, d.h. die verschiedenen Funktionen ergeben sich über die Modifizierung und Spezifizierung der relationalen Basissemantik.

3.) Die relationale semantische Basis besteht im wesentlichen aus zwei Elementen: erstens aus einer allen Partikeln gemeinsamen indexikalischen Struktur und zweitens aus den die Einzellexeme unterscheidenden semantischen Merkmalen (ihrem denotativen Gehalt). Der verweisende Anteil verknüpft die Äußerung, in der sich die Partikel befindet, mit einem weiteren Element außerhalb der Äußerung. Der denotative Gehalt der Partikel qualifiziert die durch die relationale Struktur hergestellte Verknüpfung, d.h. er bringt zum Ausdruck, in welcher Hinsicht etwas verknüpft wird.

4.) Die verschiedenen modalen und diskursiven Funktionen lassen sich aufgrund der Art des Bezugselementes unterscheiden, d.h. sie ergeben sich daraus, daß die Verknüpfung Entitäten verschiedener linguistischer und kommunikativer Ebenen betrifft. Die Modalpartikelfunktion verknüpft propositionale Entitäten, d.h. sie strukturiert die inhaltlich-argumentative Ebene der Kommunikation. Die Diskursfunktionen der Partikeln strukturieren code-, partner- und handlungsbezogene Einheiten.

Unsere Materialgrundlage ist ein Korpus aus Instruktionsdialogen (Sagerer et al. 1994). Es handelt sich um natürliche spontansprachliche Dialoge, die im Szenario einer Konstruktionsaufgabe aufgenommen wurden. Bei teilweise blockierter, eingeschränkter oder freier Sicht auf den Kommunikationspartner war die Aufgabe des einen Sprechers (Instrukteur (I)), seinem Partner (Konstrukteur (K)) beizubringen, ein Spielzeugflugzeug zusammenzubauen. Die Aufnahmen wurden auf DAT mit Studierenden der Universität Bielefeld in einem Büroraum (nicht in einem schallisolierten Raum) durchgeführt, so daß eine relativ natürliche Gesprächsatmosphäre entstehen konnte.

Männliche und weibliche Sprecher sind zu gleichen Teilen vertreten. Ein systematischer Einfluß der Variable Sicht auf die Verwendung von Diskurspartikeln konnte nicht gefunden werden. Die Dialoge werden daher im folgenden als ein Korpus betrachtet.

Das Korpus umfaßt 22 Dialoge mit insgesamt 25914 Wörtern, davon 1826 verschiedene Lexeme (Fischer \& Johanntokrax 1995). Der Anteil von Diskurspartikeln an der Gesamtwortzahl liegt bei $9,8 \%$, der Anteil an den 150 häufigsten Lexemen bei $9,3 \%$. 


\section{Funktionale Differenzierung der Partikellexeme}

Die Kategorisierung und Abgrenzung der Partikeln ist, wie auch immer man diese Wortgruppe definieren mag, ein viel diskutiertes und ungelöstes Problem. Für unsere Aufgabenstellung ist diese Frage jedoch nicht von Belang, und wir wollen nur festhalten, daß die hier zu verhandelnden vier Lexeme ja, aber, auch, doch unbestritten zum Kernbereich der Partikelklasse im Deutschen gehören und sich, wie fast alle Partikeln, durch Polyfunktionalität auszeichnen. ${ }^{1}$ Wir sprechen hier von Polyfunktionalität und nicht, wie es oft getan wird, von "Homonymie", da damit die Existenz mehrerer Zeichen mit zufällig gleichem Zeichenkörper postuliert und der semantische Zusammenhang übergangen wird. Stattdessen gehen wir von einer in allen Funktionen konstant bleibenden relationalen Semantik für jedes der Lexeme aus.

Die verschiedenen Funktionen korrespondieren mit bestimmten formalen und semantischen Kriterien, die in Helbig/Buscha (1986:475ff.) ausführlich erörtert werden. Für die Funktion als Modalpartikeln sind folgende Kriterien relevant: Modalpartikeln sind nicht flektierbar, nicht satzgliedfähig und an die Position im Mittelfeld gebunden, also an die Stelle nach dem finiten Verb und vor den infiniten verbalen Elementen (Abraham 1990, 1991). Modalpartikeln "modifizieren" den ganzen Satz, d.h. sie haben Satzskopus. Weiterhin ist festzustellen, daß die meisten Modalpartikeln auf bestimmte Satzarten beschränkt sind. So tritt laut Gelhaus (1995:372) ja nur in Aussagen auf, denn nur in Fragen (vgl. Ickler 1994:377). Doch andererseits ist sehr flexibel und nur in Entscheidungsfragen mit Frageintention ausgeschlossen (Helbig/Buscha 1986:487).

Diese Kriterien werden üblicherweise zur Abgrenzung der Modalpartikeln von konjunktionalen und adverbialen Funktionen verwendet, und sie werden auch hier als Ausgangsbasis für eine Einteilung der verschiedenen Funktionen der vier Partikeln gebraucht. Dies sei anhand einiger Beispiele aus dem Korpus kurz illustriert:

Für aber wird üblicherweise unterschieden der Gebrauch als adversative, nebenordnende Konjunktion wie in (1), der Gebrauch als Adverb wie in (2) und der Gebrauch als Modalpartikel wie in (3):

(1) ich kann wohl die Rhombe mit der Schraube und dieser einen Schiene zusammenmachen, aber dann habe ich die immer noch nicht an dem ganzen Gerät

(2) also den mit fünf und den mit drei, und zwei Löcher überlappen sich, zeigen aber in die gleiche Richtung ...

(3) das ist aber keine gute Konstruktion.

1 Vgl. Helbig/Buscha (1986:475ff.), die die Partikeln ausführlich behandeln und eine relativ geschlossene Klasse von insgesamt etwa 40 Wörtern ansetzen. Die 15 häufigsten Modalpartikeln sind aber, auch, bloß, denn, doch, eben, eigentlich, etwa, halt, ja, mal, nur, schon, vielleicht, wohl (nach Gelhaus 1995:371 und Helbig/Buscha 1986:487ff.). Die häufigsten Diskurspartikeln in den hier untersuchten Dialogen sind dagegen: ach, äh, ähm, also, gut, hm, ja, nee, nein, oh und okay (Fischer \& Johanntokrax 1995). 
Ähnliches gilt für auch: In (4) fungiert auch in Verbindung mit und als Konjunktion, in (5) ist auch Adverb, in (6) Modalpartikel.

(4) und dann halt vorne reinsteckst und auch zuschraubst mit einer kurzen gelben Schraube

(5) und die mit Kerbe brauchst du auch

(6) ja, das ist auch erstmal so richtig

Wie Beispiel (1) zeigt, ist die Konjunktion aber nicht satzgliedfähig, sondern steht vor der ersten Satzgliedposition und bewirkt eine adversative Anknüpfung des zweiten Teilsatzes an den ersten. An den Beispielen (5) und (6) wird deutlich, wie die Modalpartikelfunktion von der adverbiellen Funktion getrennt werden kann: in der adverbiellen Funktion ist eine Frontierung möglich, also z.B. für (5) auch brauchst du die mit Kerbe. Die Modalpartikel auch in (6) dagegen kann nicht frontiert werden. Sie ist nicht satzgliedfähig, nicht erststellenfähig und ans Mittelfeld gebunden.

Diesselben Kriterien gelten für doch, das in (7) adverbiell gebraucht ist, während es in Satz (8) als Modalpartikel vorliegt (als Konjunktion ist doch im Korpus nicht belegt).

(7) ja dann war es nee nee dann ist es mit dann war das weiße Ding doch falsch rum

(8) ist doch ein Klacks für Dich

Die Festlegung auf die Mittelfeldposition trennt die Modalpartikeln nicht nur von den Konjunktionen und Adverbien, sondern auch von den Diskurspartikeln. Letztere sind nicht in die syntaktische Struktur des Satzes integriert, und viele Diskurspartikeln können - in unterschiedlichen Funktionen (Fischer 1996) - an verschiedenen Positionen eingesetzt werden, sind also meist nicht an ein bestimmtes Syntagma gebunden. Zum Beispiel kann $j a$ als Diskurspartikel äußerungsinitial und -final aufreten, häufig auch in zweiter Position oder allein. Dies zeigen die Beispiele (9) bis (12).

(9) ja und damit da von die zweite Stelle verschraubst Du jetzt mit der gelben runden Schraube

(10) dieser <pause> diese Siebener ja die waren nicht beide auf einer Seite?

(11) und dann kommt der Querflïgel, ja?

(12) I: hast Du?

$\mathrm{K}: \mathrm{ja}$.

Diese stellungsgebundene Polyfunktionalität trifft auf die meisten Diskurspartikeln zu; eine Ausnahme bildet z.B. ne, das nur äußerungsfinal auftritt. Die Positionen, die die Diskurspartikeln einnehmen können, sind jedoch, im Gegensatz. zu den Adverbien und Modalpartikeln, immer ohne direkte grammatische Bindung an das Satzgefüge.

Schließlich trennt die oben genannte Skopuseigenschaft der Satzmodifizierung die Modalpartikeln von anderen Partikeln, wie den Gradpartikeln und den Fokuspartikeln, die einzelne Satzglieder modifizieren, also z.B. gradieren oder hervorheben (Gelhaus 
1995:370ff.). ${ }^{2}$ So modifiziert auch in (13) das temporale Adverbial schon wieder, in (14) modifiziert es die Nominalphrase dieses Fünflöcherige:

(13) nee nee so ist es auch schon wieder falsch <pause 18 sec. > <laut stöhnt> ich weiß auch nicht.

(14) auch dieses Fünflöcherige direkt auf diesen Klotz legen, oder?

In prototypischen Fällen erlauben die genannten Kriterien, die hier nur skizzenhaft erläutert wurden, durchaus eine klare Abgrenzung der verschiedenen Funktionen, oder genauer: die Berechtigung des Ansetzens verschiedener Kategorien ergibt sich erst durch die Anwendbarkeit solcher Kriterien. Allerdings führen die genannten unterscheidenden Merkmale nicht in jedem Fall zu einer eindeutigen Differenzierung der verschiedenen Funktionen. Da z.B. Adverbien ebenfalls im Mittelfeld stehen können, ergibt sich oft eine Ambiguität zwischen Adverb und Modalpartikel (vgl. Helbig/Buscha 1986:340). Ein weiterer Überschneidungsbereich zeigt sich in Beispiel (15), das einen Auszug aus dem Korpus bietet, wo nicht sicher entschieden werden kann, ob auch und aber als Diskurspartikeln mit Segmentierungsfunktionen in derselben Funktion wie also auftreten, oder ob es sich um Adverbien, bzw. Konjunktionen handelt:

(15) dann schraubst Du von unten an den Klotz einen einen Dreier <pause $>\underline{\text { auch }}$ mit einer roten Schraube und zwar so, daß sie zum öh zur oberen Leiste $90 \mathrm{Grad}$ ergibt, aber mit der letzten mit dem letzten Loch also nicht in der Mitte, genau.

Auf diese Fälle der kategorialen bzw. funktionalen Ambiguität werden wir später noch genauer eingehen. Gerade in dialogischen Texten sind derartige Übergangsfälle und Grenzverwischungen ausgesprochen zahlreich. Unseres Erachtens ist die Existenz dieses Kontinuums zwischen den prototypischen Zentren der einzelnen Kategorien ein stichhaltiges Argument für das hier vorgebrachte Postulat einer gemeinsamen semantischen Grundstruktur, die sozusagen als kleinster gemeinsamer Nenner die funktionale Variablität überhaupt erst ermöglicht. Bevor wir auf die Übergänglichkeit der Partikeln eingehen, wollen wir in den folgenden beiden Abschnitten die Semantik der modalen und der interaktiven Funktionen darstellen.

\section{Relationale Basisstruktur von Diskurs- und Modalpartikeln}

Unsere These, daß die Modalpartikeln und die Diskurspartikeln im heutigen Deutsch zumindest im Kernbereich eine "ordentliche" grammatische Funktion

2 Helbig/Buscha (1986:477) verzichten hier auf eine Subklassifikation und teilen die gesamte Gruppe der Partikeln in "solche Partikeln, bei denen die kommunikative Funktion dominiert" - das entspricht den Modalpartikeln - und "solche Partikeln, bei denen die semantische Funktion dominiert" (477), "die ein Wort im Satz näher bestimmen, erläutern, spezifizieren oder graduieren" (476); in diese Gruppe fallen u.a. die Gradpartikeln. Zur Abgrenzung der Modalpartikeln von den "scalar particles" s.a. Abraham (1991:243ff.). 
aufweisen, die auf ein abstraktes semantisches Basisschema zurückzuführen ist, ist keineswegs selbstverständlich. Was die Modalpartikeln betrifft, um die es im folgenden Abschnitt zunächst geht, so lautet eine ihrer inzwischen etwas altmodischen Benennungen "Würzwörter", und wenn man unter Würze zwar Angenehmes, aber Überflüssiges, unter Grammatik dagegen das absolut notwendige Grundgerüst einer Sprache versteht, dann wird sofort augenfällig, daß den Modalpartikeln unter diesem Namen keinerlei ernsthafte grammatische Funktion zugestanden wurde. Selbst Eisenberg (1994:206) nennt in seiner Grammatik die Partikeln "die Zaunkönige und Läuse im Pelz der Sprache" und schließt sich expressis verbis der Tradition des Übergehens dieser Wörter an. Gelhaus in der Duden-Grammatik hält fest, daß die Partikeln " - anders als Präpositionen und Konjunktionen - keine grammatische Funktion haben" (1995:369). Selbst Helbig/Buscha (1986:479), die die Partikeln ausführlich behandeln, treffen eine Unterscheidung zwischen den "kommunikativpragmatischen Funktionen" der Modalpartikeln und "eigentlichen" grammatischen Funktionen der Sprache, an denen die Modalpartikeln ihrer Auffassung nach offenbar keinen Anteil haben.

Nun hat zwar die Partikelforschung - ausgehend von Weydt 1969 - in den letzten Jahren einen enormen Aufschwung genommen, doch trotz der vielschichtigen Forschungslage, die hier nicht referiert werden kann (einen Forschungsbericht gibt Ickler 1994), ist der Frage ihrer konstitutiven grammatischen Funktion, soviel uns bekannt, bisher kaum Aufmerksamkeit geschenkt worden. Der grammatische Status der Partikelfunktionen muß also im folgenden noch erwiesen werden. Dieser Frage werden wir uns zunächst für die Modalpartikeln und anschließend für die Diskurspartikeln widmen.

\subsection{Modalpartikeln}

Worin also besteht die hier postulierte grammatische Bedeutung und Funktion der Modalpartikeln? Die Beschreibungen in den Grammatiken sind meist allgemein gehalten: Eisenberg (1994:206) hält fest, daß die Modalpartikeln "den Inhalt des Satzes auf die Sprechsituation [...] beziehen". Gelhaus (1995:371) schreibt dazu:

"Mit Modalpartikeln drückt der Sprecher eine Annahme, Erwartung oder innere Einstellung aus, oft in der Absicht, daß sie der Hörer teilt. Der Sprecher kann mit Modalpartikeln seinen eigenen Aussagen eine bestimmte (subjektive) Tönung geben, er kann damit aber auch auf vorausgegangene Äußerungen Bezug nehmen und Zustimmung, Ablehnung, Einschränkung, Erstaunen, Interesse anzeigen."

Helbig/Buscha (1986:476) sprechen davon, daß die Modalpartikeln

"Indikatoren für bestimmte Sprechhandlungen [sind oder dazu dienen,] die Äußerung im konversationellen Kontext zu verankern, den Sprechakt im Sinne des Sprechers zu modifizieren, den Interpretationsprozeß des Hörers und die Interaktion zu steuern bzw. - allgemein gesprochen - die Äußerung und den Sprechakt auf die Gegebenheiten der Interaktion zu beziehen." 
Derart vielschichtige Funktionsbeschreibungen lassen zunächst Zweifel aufkommen, ob man hier wirklich eine einheitliche grammatische Grundfunktion finden kann. Ein gemeinsames Merkmal aller Modalpartikeln ist jedoch aus diesen Beschreibungen abzulesen, nämlich ihr abstrakter relationaler Gehalt, also ihre verweisende, indexikalische Funktion, die auch schon mehrfach bemerkt wurde (Henschtel 1986, Petri 1995).

Diese indexikalische Funktion ist nun eine genuin grammatische Funktion, ja ein wesentliches Kennzeichen aller grammatischen Kategorien. So markieren die grammatischen Kategorien Tempus und Modus am Verbum die Verortung des dargestellten Sachverhalts in bezug auf den Sprecher, d.h. auf die deiktische Origo (Bühler 1982 [1934]:102ff., Diewald 1991:27ff.). Diese ist der Ausgangs- und Bezugspunkt, von dem aus das Dargestellte eingeordnet wird. Zum Beispiel hat die deiktische grammatische Kategorie Präteritum die Funktion, den dargestellten Sachverhalt in bezug auf die Sprechzeit temporal als vergangen, d.h. entfernt von der Sprechzeit, einzuordnen. In Satz (16)

(16) Sie machte einen Umweg durch den Park

wird also durch das Präteritum der Sachverhalt Sie macht einen Umweg durch den Park in bezug auf die temporale Dimension als 'entfernt vom Sprecher' (im Gegensatz zum Präsens) gekennzeichnet. Auch die textverweisende Funktion der Proformen basiert auf dieser indexikalischen Struktur. Die anaphorische Proform sie im folgenden Satz

(17) Die Katze wollte ins Haus zurück. Sie sprang auf das Fensterbrett und drückte sich an die Scheibe,

verweist zurück auf die Nominalphrase die Katze, die das Bezugselement für die Proform darstellt und deren Gehalt von der Proform vermittelt übernommen wird. Den Konjunktionen liegt ebenfalls eine verweisende Komponente zugrunde, die bereits anhand von Beispiel (1) erläutert wurde. Die Konjunktion aber weist auf den vorausgehenden Satz zurück, mit dem sie den angeschlossenen zweiten Satz verknüpft:

(1) Ich kann wohl die Rhombe mit der Schraube und dieser einen Schiene zusammenmachen, aber dann habe ich die immer noch nicht an dem ganzen Gerät

Die genannten grammatischen Zeichen bzw. Kategorien (es könnten weitere angeführt werden) haben also eine indexikalische Komponente, die darin besteht, daß das grammatische Zeichen die von ihm modifizierte Einheit mit einem Bezugselement verbindet bzw. auf ein Bezugselement zurückverweist. Diagramm (18) gibt eine grobe Skizze dieses Prozesses, die zwar stark vereinfacht, aber für unsere Zwecke ausreicht:

(18) Bezugselement $\leftarrow$ (grammatisches Zeichen \& vom grammatischen Zeichen modifizierte Einheit)

Für die verschiedenen grammatischen Funktionen kann diese Skizze spezifiziert werden. Für Tempusflexive z.B. hat sie folgende Form:

(19) Sprechzeit $\leftarrow$ (Tempusflexiv \& Sachverhaltsdarstellung)

Für anaphorische Proformen wie sie in (17) kann Schema (20) angeführt werden: 
(20) Textuell vorausgehende Nominalphrase $\leftarrow$ (Pronomen \& Satzgliedfunktion)

Die konjunktionale Variante der grammatischen Relationsstruktur ist in (21) illustriert:

(21) Sachverhaltsdarstellung $1 \leftarrow$ (Konjunktion \& Sachverhaltsdarstellung 2)

Die Schwierigkeit, die analoge verweisende Funktion bei den Modalpartikeln exakt zu erfassen, ergibt sich daraus, daß die grammatische, relationale Funktion der Modalpartikeln weder im Bereich der Darstellungsfunktion des Satzes liegt (wie z.B. bei den Proformen), noch im Bereich der Verknüpfung von Teilsätzen (wie bei Konjunktionen), noch im Bereich der deiktischen Verankerung des Dargestellten in bezug auf die Sprechzeit (wie bei den Tempusflexiven). Die Modalpartikeln kodieren also keine syntaktischen und semantischen Relationen zwischen Satzgliedern oder Teilsätzen, noch bringen sie eindeutig deiktische Relationen zwischen Zeichenbenutzer und Sachverhaltsdarstellung zum Ausdruck. Kurz gefaßt, das Problem der Explizierung der grammatischen Funktion der Modalpartikeln liegt in der Bestimmung ihres Bezugselements, also des Elements, auf das sie als zur Interpretation der Äußerung relevant zurückverweisen.

Dieses Bezugselement und damit die relationale Funktion der Modalpartikeln läßt sich erfassen, wenn man eine modalpartikelhaltige Äußerung mit der entsprechenden Äußerung ohne Modalpartikel kontrastiert. Beispiel (3) wieder aufgreifend ergibt sich also ein Kontrast zwischen (3) und (22):

(3) Das ist aber keine gute Konstruktion.

(22) Das ist keine gute Konstruktion.

Der Vergleich der beiden Sätze zeigt, daß aber ein Bedeutungselement hinzufügt, das man zunächst als "Verweis auf ein Auseinanderfallen, einen Gegensatz, von Sprechererwartung und vom Sprecher wahrgenommener und dargestellter Realität" kennzeichnen könnte. Während Satz (22) eine bezüglich modaler Inhalte neutrale Sachverhaltsdarstellung ist, drückt (3) zusätzlich aus, daß der Sprecher etwas anderes erwartet bzw. vorausgesetzt hatte als das, was er nun in der partikelhaltigen Äußerung als Sachverhalt darstellt. Im konkreten Fall (3) bringt die Partikel zum Ausdruck, daß der Sprecher die Proposition daß das eine gute Konstruktion ist als vorgegeben betrachtet, daß die aktuelle Situation jedoch von der Vorgabe abweicht.

Die Funktion der Partikel ist es also, die Äußerung mit einem Sachverhalt zu verknüpfen, den der Sprecher als relevant, als im Raum stehend, betrachtet und auf den er die partikelhaltige Äußerung bezieht. Damit ist die zentrale Funktion aller Modalpartikeln erfaßt: indem Modalpartikeln einen Verweis auf Vorgegebenes enthalten, markieren sie die Äußerung, in der sie stehen, als nicht-initial.

Der vorgegebene Sachverhalt ist typischerweise nicht im Vortext genannt, die Modalpartikeln sind also keine textverknüpfenden Elemente im engeren Sinn. Damit ist gleichzeitig gesagt, daß der Inhalt der Vorgabe, auf die der Sprecher Bezug nimmt, erst durch die partikelhaltige $\ddot{u}$ ßerung selbst explizit wird. 
Der vorgegebene Sachverhalt ist daher, was die Kernproposition betrifft, mit dem geäußerten Sachverhalt identisch: im obigen Beispiel enthält sowohl die Vorgabe als auch die Äußerung die Proposition daß das eine gute Konstruktion ist. Durch die partikelhaltige Äußerung wird diese Proposition thematisiert, d.h. es wird gekennzeichnet, daß es um die Proposition daß das eine gute Konstruktion ist geht. Durch die Semantik der Partikel selbst (und durch die Negation) wird eine vom Sprecher gesetzte Modifikation der Vorgabe ausgedrückt, in diesem Fall ein Gegensatz zwischen der vorgegebenen Proposition und dem tatsächlichen Sachverhalt.

Die Partikel verweist also auf eine noch nicht vertextete Vorgabe; dies ist ihre indexikalische Komponente, die allen Modalpartikeln gemeinsam ist. Gleichzeitig wird die Vorgabe durch die Partikel modifziert bzw. qualifiziert; dies ist die denotative Komponente der Partikeln, die je nach Partikellexem verschieden ist.

Die vorgegebene Proposition bezeichnen wir im folgenden als den pragmatischen Prätext. Von Prätext sprechen wir deshalb, weil es sich um eine inhaltliche bzw. propositionale Einheit - nicht um emotionale oder expressive Komponenten - handelt, von pragmatischem Prätext sprechen wir deshalb, weil diese Proposition typischerweise vorher nicht versprachlicht ist.

Damit vertreten wir eine Auffassung von der Funktion der Partikeln, die der von Ickler 1994 nahekommt. Er argumentiert überzeugend gegen eine Funktionsbestimmung im Sinne "illokutiver Indikatoren" (Helbig/Buscha 1986:480) ebenso wie gegen eine Bestimmung im Sinne emotional-expressiver Sprechereinstellung: ${ }^{3}$

"Die Modalpartikeln passen bestimmte Äußerungen in größere, grundsätzlich dialogisch zu interpretierende Textzusammenhänge ein. Sie haben daher [...] viel mehr mit der natürlichen Logik des Gesprächs zu tun als mit der 'expressiven' oder 'emotionalen' Seite der Kommunikation" (1994:377f.).

Weiter beschreibt Ickler (1994:404) die Partikeln in ihrer unbetonten Normalfunktion als "eine Art von Kommentar zu der jeweiligen Äußerung, in der sie vorkommen, einen Kommentar, der die logische oder rhetorische Einordnung der Äußerung in den Kommunikationszusammenhang betrifft". Auch Doherty (1985:15), die die Partikeln zu den "positionalen Ausdrucksmitteln" zählt, bezieht sich auf deren relationale Funktion; desgleichen Hentschel (1986:31), die von der "metakommunikativen Deixis" der Modalpartikeln spricht. Abraham (1990:129) bestimmt die Leistung der Modalpartikeln als "textdeiktische, textkohärenzschaffende", was zwar keinen Zweifel an ihrem relationalen Charakter läßt, was aber dann zu kurz greift, wenn man unter "Text" nur die versprachlichten Anteile der Kommunikation zusammenfaßt. Die relationale Funktion der Modalpartikeln betrifft nicht den Text als sprachlich realisierte Größe, sondern implizite Vorgaben. 
Die verweisende Struktur der Modalpartikeln ist in Diagramm (23) wiedergegeben, was die Parallelität zu den übrigen indexikalischen grammatischen Kategorien deutlich macht (vgl. (18)-(21)):

(23) Pragmatischer Prätext $\leftarrow$ (Partikellexem \& Äußerung im Skopus der Modalpartikel)

Der Unterschied zu der indexikalischen Funktion der Konjunktionen verdeutlicht die Eigenheit der Modalpartikeln: Konjunktionen beziehen sich auf ein sprachlich realisiertes Element des Vortextes, die Partikeln auf ein nichtrealisiertes. Konjunktionen verbinden typischerweise zwei Einheiten mit unterschiedlichem propositionalem Gehalt, also z.B. zwei Sätze mit je einer Sachverhaltsdarstellung: sie drücken die Relation zwischen zwei verschiedenen Propositionen aus, also z.B:

(24) Sie wollte telefonieren,

Proposition 1 aber

Konjunktion sie hatte kein Kleingeld.

Proposition 2

Modalpartikeln dagegen verbinden zwei im Kern gleiche Propositionen, sie drücken ihre Modifizierung aus.

(25) (nicht versprachl.: daß das eine gute K. ist) das ist aber keine gute Konstruktion

pragmatischer Prätext: Proposition $1 \leftarrow$ (Modalpartikel \& modifizierte Proposition 1')

Nachdem nun das indexikalische Grundschema der Modalpartikeln erörtert ist, ist zu fragen, wie ihre spezifische denotative Komponente (also das adversative Element von aber, das affirmierende Element von $j a$ etc.) in die Beschreibung eingebracht werden kann. Dazu verwenden wir ein Schema, das sowohl den pragmatischen Prätext als auch die relevante Situation, die den Sprecher zu einer Modifizierung des pragmatischen Prätextes veranlaßt, darstellt. Wir lehnen uns hierbei an Wierzbickas $(1986,1991)$ Konzept der universalen semantischen Metasprache an, ohne jedoch deren Postulat zu übernehmen, daß die für die Bedeutungsbeschreibung verwendeten Lexeme universale semantische Primitive repräsentieren. Unsere Bedeutungsexplikationen sind also tentativ und sollten als Vorschläge aufgefaßt werden. Das Grundschema ist in (26) gegeben:

(26) Grundschema zur Bedeutungsbeschreibung der Modalpartikeln

pragmatischer Prätext

relevante Situation:

$\rightarrow$ Äußerung: im Raum steht: Proposition

Sprecherbewertung bezüglich der im Raum stehenden Proposition

Modifizierte Proposition mit Partikel

Für eine Beschreibung der Bedeutung von aber in Beispiel (3)

(3) Das ist aber keine gute Konstruktion.

wird das Grundschema folgendermaßen konkretisiert:

(27) Bedeutungsschema der Modalpartikel aber 
pragmatischer Prätext:

relevante Situation:

$\rightarrow$ Äußerung: im Raum steht: daß das eine gute Konstruktion ist.

ich denke: das ist keine gute Konstruktion

Das ist aber keine gute Konstruktion!

Der Satz mit Modalpartikel ist quasi das Ergebnis der Einbeziehung und Komprimierung des pragmatischen Prätextes in die aktuelle Äußerung. Die Modalpartikel weist auf den pragmatischen Prätext zurück und bringt ihn mit der geäußerten Sachverhaltsdarstellung in Beziehung. Die Formulierung "im Raum steht" soll anzeigen, daß es sich um einen vom Sprecher als vorgegeben betrachteten Sachverhalt handelt, auf den er mit seiner Äußerung Bezug nimmt. Dabei legen die Partikeln typischerweise nicht fest, von wem diese Vorgabe stammt bzw. woher die Information kommt, die im Raum steht. Zwar wird in partikelhaltigen Äußerungen häufig auf dem Hörer bekannte Vorgaben Bezug genommen, bzw. es wird unterstellt, daß der Hörer diese Vorgaben macht, jedoch ist dies keine Bedingung zur Verwendung der Partikeln, und nicht selten beziehen sich die Partikeln auf generell gültige Standardvoraussetzungen. Das heißt, der Sprecher nimmt allgemein auf eine Einheit Bezug, die seiner Meinung nach im Raum steht bzw. vorgegeben ist, ohne sich festzulegen, wer diese Vorgabe macht. Durch die partikelhaltige Äußerung wird ausgedrückt, daß der Sprecher diese Vorgabe aufnimmt und seine Äußerung auf sie bezieht, also keinen initialen Gesprächszug durchführt.

\subsection{Diskurspartikeln}

Unter Diskurspartikeln verstehen wir im folgenden Lexeme mit Strukturierungseigenschaften (Gülich 1970), die nicht flektierbar und nicht in das Satzgefüge integrierbar sind, und die sich inhaltlich auf mentale Vorgänge beziehen (Schourup 1983). Diese Klasse umfaßt die Klasse der traditionellen Interjektionen (Wierzbicka 1992), die der Gliederungssignale (Willkop 1988), sowie die Häsitationsmarker, z.B. äh und $\ddot{a} h m$.

Wir gehen davon aus, daß die Diskurspartikeln ebenfalls eine grammatische Funktion besitzen. Für die Diskurspartikeln einen grammatischen Status anzunehmen, scheint einerseits noch problematischer als für die Modalpartikeln, da erstere nicht in die syntaktische Struktur integriert sind und auch keine offensichtlichen syntaktischen Bindungen zur Satzstruktur aufweisen. Andererseits hebt bereits Gülich (1970) hervor, daß Gliederungssignale nur in Hinblick auf die Dialogstruktur überhaupt erst als Klasse erkennbar sind, d.h. die Diskurspartikeln sind ihrem Wesen nach relational auf den Diskurs bezogen. Auch Wilkins (1992) stellt Interjektionen als wesentlich indexikalisch dar. Es gilt also auch hier, die Bezugselemente, die für die Klasse der Diskurspartikeln charakteristisch sind, zu identifizieren, um die Zuschreibung eines grammatischen Status zu erlauben, wobei sich die Dialogstruktur bereits als ein zentrales Bezugselement für die Diskurspartikeln darstellt. 
Die These ist hier nun, daß sich die verschiedenen Funktionen von Diskurspartikeln wie die der Modalpartikeln durch eine gemeinsame relationale Basissemantik bestimmen lassen, die durch Merkmale der Diskurssituation ergänzt wird. Im Gegensatz zu der für die Klasse der Modalpartikeln charakteristischen Rolle des pragmatischen Prätextes als Bezugsgröße beziehen sich die Diskurspartikeln auf verschiedene Aspekte des Kommunikationssystems. Das, was bei den Modalpartikeln die Rolle des pragmatischen Prätextes erfüllt, kann bei den Diskurspartikeln sowohl durch den außersprachlichen Kontext, z.B. die Handlungssituation, gegeben sein als auch durch versprachlichte Propositionen, wie beispielsweise im Fall von Antwortpartikeln. Die Polyfunktionalität der Diskurspartikeln kommt dabei dadurch zustande, daß sich die relationale Basissemantik desselben Lexems auf verschiedene kommunikative Ebenen beziehen kann.

Bei der Beschreibung der Diskurspartikeln als Elemente mit relationaler Basissemantik ergeben sich einige Schwierigkeiten, die durch die Eigenschaften der Diskurspartikeln bedingt sind: Interjektionen und Antwortpartikeln sind grundsätzlich als satzwertig anzusehen, aber auch Gliederungssignale und Häsitationen können eigenständig verwendet werden. In der Bedeutungsexplikation der Modalpartikeln ist die relevante Situation, zu der der pragmatische Prätext in Beziehung tritt, jeweils durch die Äußerung gegeben, in der die Partikel verwendet wird. Bei Diskurspartikeln, die eigene Äußerungen konstituieren, ist ein solcher Kontext nicht gegeben. Er muß also noch zusätzlich zu dem Bezugselement erschlossen werden. Aber auch für Diskurspartikeln, die beispielsweise innerhalb von größeren intonatorischen Einheiten geäußert werden und die somit einen direkten Äußerungskontext aufweisen, ist die relevante Situation nicht automatisch gegeben, da sich Diskurspartikeln auf verschiedene Elemente der Kommunikationssituation beziehen können, die nicht explizit thematisiert werden. So konstituieren die Diskurspartikeln sozusagen einen Nebenschauplatz neben dem, was explizit geäußert wird.

Eine Ausnahme bilden hier die Antwortpartikeln, die sich auf die propositionale Information aus der Vorgängeräußerung beziehen. Für alle anderen Funktionen der Diskurspartikeln gilt, daß sie sich auf nicht-thematisierte kommunikative Ebenen beziehen, wobei erschwerend hinzukommt, daß meist mehrere Aspekte gleichzeitig involviert sind.

Wir gehen davon aus, daß diese Bedeutungen sprachlich erfaßt werden können, auch wenn diese Aspekte meist mentale Zustände des Sprechers beschreiben, die nicht zu unserem gewöhnlichen Gesprächsstoff gehören. Wie für die Bedeutungen der Modalpartikeln werden diese Bedeutungsaspekte in natural semantic metalanguage (NSM) (Wierzbicka 1986) formuliert, wobei wir annehmen, daß NSM ohne Probleme von der englischen Sprache in die deutsche übertragen werden kann. Trotz einiger Vorbehalte gegen NSM (vgl. Fischer demn.) halten wir NSM für eine geeignete Metasprache, sich den Bedeutungen der Diskurspartikeln anzunähern. Die Bezugselemente der Diskurspartikeln können daher ebenfalls sprachlich formuliert werden. Da nun verschiedene Aspekte des Kommunikationssystems die Bezugselemente der 
Diskurspartikeln bilden, d.h. sie sich auf Merkmale der Gesprächssituation beziehen, sprechen wir im folgenden vom pragmatischen Kontext als dem Bezugselement der Diskurspartikeln.

Es werden nun drei kommunikative Ebenen, auf die sich die Diskurspartikeln beziehen können, exemplarisch dargestellt.

Das Antwortsignal ja z.B. bezieht sich auf die propositionale Information der Vorgängeräußerung, mit der Übereinstimmung ausgedrückt wird. Im Schema, das wir an den Modalpartikeln demonstriert haben, läßt sich die relationale Bedeutung von $j a$ als Antwortsignal folgendermaßen verdeutlichen:

(28) Bedeutungsschema der Antwortpartikel ja in Frage-Antwort-Paaren:

Äußerungskontext:

Du fragst: hast $D u$ ?

relevante Situation:

ich habe

$\rightarrow$ Äußerung ja

Das Bezugselement der von ja ausgedrückten Bedeutung der Übereinstimmung ist hier also eine versprachlichte Äußerung über einen Zustand in der Welt, die als Frage, aber auch als Aussage, gefaßt sein kann. Bei der Antwortpartikel doch konstituiert dagegen keine Übereinstimmung sondern ein Widerspruch die Relation zwischen Äußerungskontext und relevanter Situation:

(29) $\mathrm{K}$ : das hält gar nicht

I: doch, du mußt die andere weiter, äh

Das Bedeutungsschema für die Antwortpartikel doch (hier in Reaktion auf eine Feststellung) kann folgendermaßen skizziert werden:

(30) Bedeutungsschema für die Antwortpartikel doch:

Äußerungskontext:

Du sagst: das hält gar nicht.

relevante Situation:

ich denke: das hält

$\rightarrow$ Äußerung:

doch.

Als Antwortpartikeln beziehen sich ja und doch also auf die propositionale Information aus dem Äußerungskontext.

Diskurspartikeln können sich aber auch auf die thematische Struktur des Dialogs beziehen wie in dem folgenden Beispiel mit aber, wo der Konstrukteur sich schon nach dem nächsten Bauschritt erkundigt:

(31) $\mathrm{K}$ : und das wird dann da so seitlich draufgeschraubt oder?

I: ja genau, aber mach das erstmal so.

Die schematische Repräsentation bezieht sich dabei auf das herrschende Thema einerseits sowie den Wunsch des Sprechers, das Thema zu wechseln, andererseits:

(32) Bedeutungsschema für aber als Themensignal:

pragmatischer Kontext:

wir reden über etwas

relevante Situation:

ich möchte über etwas anderes reden 
Diskurspartikeln können weiterhin zur Verständnis- und Zustimmungsabsicherung gebraucht werden. In Äußerung (33) wird beispielsweise ein Signal über die grundsätzliche Übereinstimmung von Sprecher und Hörer elizitiert. Die Semantik von $j a$ in dieser Verwendung könnte daher formuliert werden als: "denken wir dasselbe?" Gleichzeitig kann mit Brown \& Levinson (1987) dem Sprecher unterstellt werden, daß die Übereinstimmung Ziel des Dialogs ist: "Ich wünsche, wir denken dasselbe". Mit der Verwendung von $j a$ und oder doch in (33) und (34) wird also eine zustimmende Rückmeldung vom Hörer erwartet:

(33) den Querflügel, ja?

(34) die gucken nicht beide auf eine Seite oder doch?

In den folgenden Bedeutungsexplikationen deutet das Verb "sagen" darauf hin, daß von dem Hörer explizite Zustimmung gefordert wird:

(35) Bedeutungsschema für $j a$ als Zustimmungsabsicherungssignal

pragmatischer Kontext:

ich sage: den Querflügel

relevante Situation:

$\rightarrow$ Äußerung ich wünsche: Du sagst: den Querflügel den Querflügel, ja?

(36) Bedeutungsschema für doch als Zustimmungsabsicherungssignal pragmatischer Kontext: ich sage: die gucken nicht auf eine Seite relevante Situation: ich frage, ob Du sagst, daß sie auf eine Seite gucken

$\rightarrow$ Äußerung die gucken nicht beide auf eine Seite oder doch?

Zusammenfassend kann gesagt werden, daß die Funktion der Diskurspartikeln in der Verankerung der sprachlichen Äußerungen in der Kommunikationssituation besteht mit dem Ziel, den Ablauf des Dialogs in der Situation zu motivieren (cf. Östman 1981).

Die Polyfunktionalität der Diskurspartikeln resultiert dabei aus der Bezugnahme auf verschiedene kommunikative Ebenen. Diese Relation wurde für die Bezugselemente Äußerungskontext, thematische Struktur und schließlich Verständigungsebene dargestellt. Trotz der verschiedenen Bezugselemente weisen die Diskurspartikeln eine lexemspezifische einheitliche relationale Grundstruktur auf, die sich für $j a$ beispielsweise als grundsätzliche Übereinstimmung kennzeichnen läßt. Das Postulat eines grammatischen Status dieser spontansprachlichen Einheiten scheint damit gerechtfertigt.

Neben dem formalen Kriterium der Stellung außerhalb der Satzstruktur kann daher zur Abgrenzung der Diskurspartikeln gegen die Modalpartikeln und Konjunktionen, bzw. Adverbien auch die Art der Bezugselemente angeführt werden, die bei den Diskurspartikeln verschiedenen Aspekten des Kommunikationssystems entstammen. 


\section{Einzelanalysen}

In diesem Kapitel steht einerseits die Bedeutungsexplikation der vier Partikeln im Mittelpunkt; es werden die lexemspezifischen Bedeutungsaspekte dargestellt, aufgrund derer die Partikeln ein geordnetes semantisches Feld bilden. Andererseits geht es um Fälle, die hinsichtlich ihrer Funktionen schwer einzuordnen sind. So gibt es zahlreiche Beispiele im Korpus, für die eine eindeutige Klassifikation als Adverb, Konjunktion, Modalpartikel oder Diskurspartikel nicht möglich scheint. Diese Vorkommen unterstützen unsere These, daß die Partikeln mit einer einheitlichen relationalen Basissemantik beschrieben werden können, da ohne eine relationale Basissemantik, die unabhängig von der jeweiligen Funktion konstant bleibt, die funktionale Ambivalenz in verschiedenen Vorkommen nicht zu erklären wäre.

\subsection{Aber}

Aber ist als Modalpartikel vorzugsweise in Äußerungen mit exklamativer Funktion zu finden, die unterschiedlichen Strukturtypen (Aussagesätze, Fragesätze) angehören können:

(3) Das ist aber keine gute Konstruktion.

(37) Ja oh das ist aber schwierig

Die Auflösung in den pragmatischen Prätext, die relevante Situation und die adversative Lexembedeutung für Satz (3) wurde oben schon durchgeführt; sie ist hier wiederholt:

(38) Bedeutungsschema der Modalpartikel aber

pragmatischer Prätext:

im Raum steht: daß das eine gute Konstruktion ist.

relevante Situation: ich denke: das ist keine gute Konstruktion

$\rightarrow$ Äußerung: Das ist aber keine gute Konstruktion!

Die Grundbedeutung von aber kann also paraphrasiert werden als 'Indizierung einer adversativen Relation zwischen pragmatischem Prätext und in der Äußerung dargestellter Situation'.

Ferner tritt aber als Modalpartikel in direktiven Sprechakten auf, wie in (39) und (40), die allerdings nur als indirekte Sprechakte direktive Funktion haben, als Strukturtyp liegt ein Aussagesatz vor:

(39) Das ist echt alles ein klappriger Kram. Mußt aber gucken, ob da auch zwei Gewinde aneinander sind.

(40) Und zwar dann in den blauen Würfel, da mußt Du aber drauf achten, der Würfel hat verschieden große Löcher. 
Auch hier bleibt die relationale Grundfunktion und die adversative Semantik erhalten. Allerdings ist das Schema in Rücksicht auf den direktiven Sprechakt abzuändern; (41) zeigt dies für Beispiel (39):

(41) Bedeutungsschema der Modalpartikel aber in direktiven Sprechakten

pragmatischer Prätext: $\quad$ im Raum steht: du mußt nicht gucken, ob...

relevante Situation:

$\rightarrow$ Äußerung ich will: du mußt gucken, ob... mußt aber gucken, ob...

Bei Äußerungen wie (39) und noch deutlicher bei (40) zeigt sich ein sozusagen schleichender Übergang zwischen der zentralen Modalpartikelfunktion von aber und seiner adverbiellen bzw. konjunktionalen Funktion. Die direktiven Sprechakte schließen nämlich an eine Vorgängeräußerung an, und obwohl diese nicht in einem im engen Sinn adversativen Verhältnis zur partikelhaltigen Äußerung steht, ist es doch ganz offenkundig, daß letztere, also die partikelhaltige Äußerung, sich in unspezifischer Form auf die vorhergehende Äußerung bezieht, während die prototypische Modalpartikel ja ohne textuellen Bezugspunkt ist. Solche ambigen Verwendungsweisen sind in den Instruktionsdialogen durchaus häufig.

Die folgenden Beispiele sind ebenfalls schwer zu interpretieren in Hinblick darauf, ob aber hier nur als Konjunktion zweier Äußerungen fungiert, von denen eine abgebrochen wurde, oder ob es hier als satzwertig zu betrachten ist, also eine eigenständige Äußerung konstituiert:

(42) ähm <pause> ja aber ähm <pause> genau

(43) Also irgendwas habe ich wahrscheinlich falsch gemacht aber

(44) I: Haben wir vorhin nicht gesagt aber

$\mathrm{K}$ : jaja nee ist klar

Wie die Reaktion des Konstrukteurs in (44) zeigt, hat aber in dieser Verwendung die Funktion, eine zustimmende Reaktion des Hörers zu elizitieren, wobei die Äußerung des Konstrukteurs sogar als explizite Zusammenfassung des fehlenden Teilsatzes aufgefaßt werden kann: obwohl es vorher nicht gesagt wurde, ist es klar gewesen. Die Häufigkeit, mit der diese Konstruktion auftritt, deutet darauf hin, daß es sich hier tatsächlich nicht um einen Abbruch handelt. Stattdessen scheint aber hier als eine Diskurspartikel verwendet zu werden, die als satzwertig anzusehen ist und sich auf einen pragmatischen Prätext bezieht, nämlich die Erwartung des Gelingens des Konstruktionsschritts trotz inexpliziter Information.

\subsection{Auch}

Auch als Modalpartikel tritt im Korpus in Aussagsätzen und Fragesätzen auf; (45) und (46) sind Beispiele für diese Verwendungsweisen:

(45) Achteck? Ach so, ja, ist auch egal.

(46) Kann ich auch so mit Händen alles? (an Versuchsleiter gerichtet) 
Der Sprecher konstatiert ein iteratives bzw. augmentatives Verhältnis zwischen pragmatischem Prätext und relevanter Situation. In eine Formel gebracht kann man die Grundbedeutung der Modalpartikel auch angeben als 'Indizierung einer augmentativen Relation zwischen pragmatischem Prätext und in der Äußerung dargestellter Situation'. Das Bedeutungsschema für Äußerung (45) ist in (47) gegeben:

(47) Bedeutungsschema für die Modalpartikel auch:

pragmatischer Prätext:

relevante Situation:

$\rightarrow$ Äußerung: im Raum steht: daß es egal ist.

ich denke: es ist egal.

Ist auch egal.

Für den Fragesatz (46) gilt das Schema analog, die illokutive Funktion ist bereits in den pragmatischen Prätext integriert (vgl. auch Ickler 1994:385):

(48) Bedeutungsschema für die Modalpartikel auch in Fragesätzen:

pragmatischer Prätext:

im Raum steht: ob ich alles so mit Händen kann

relevante Situation: ich frage: kann ich so mit Händen alles

$\rightarrow$ Äußerung: Kann ich auch so mit Händen alles?

In einigen Verwendungsweisen der Partikel auch in den Instruktionsdialogen wird deutlich, daß der pragmatische Prätext bzw. das Bezugselement durchaus einen Aspekt der aktuellen Handlungs- oder Wahrnehmungssituation erfassen kann. Die Bedeutung von auch ist dann paraphrasierbar als 'Feststellung der Übereinstimmung (d.h. iteratives bzw. augmentatives Verhältnis) von zwei Situationen'. So wird in Beispiel (49) ausgedrückt, daß beide Sprecher dasselbe sehen, in (50) bezieht sich die Übereinstimmung auf die beiden möglichen Perspektiven, unter denen das Spielzeugflugzeug betrachtet werden kann. In Beispiel (51) dagegen bezieht sich auch als Adverb auf eine vorhergegangene Situation.

(49) sehe ich auch gerade

(50) das ist bei mir auf der rech/ich kann es auch umdrehen

(51) so stimmt es ja auch schon wieder nicht

Im folgenden Beispiel wird auch mit Segmentierungsfunktion gebraucht:

(52) hast Du die runde, auch gelb?

Die Bedeutung von auch in dieser Äußerung kann sich entweder auf einen vorhergegangen Bauschritt beziehen, in dem bereits eine gelbe Schraube verwendet wurde; hier wird diese zusätzliche Spezifikation als Apposition eingefügt. Oder auch bezieht sich auf die sprachliche Erweiterung selbst, in dem Fall wäre diese Verwendung von auch als Diskurspartikel zu sehen. Für auch läßt sich daher ebenfalls ein Bedeutungskontinuum für die verschiedenen Wortarten Modalpartikel, Adverb und Diskurspartikel aufzeigen. 


\subsection{Doch}

Doch als Modalpartikel ist sehr flexibel und - wie schon erwähnt - nur beim Strukturtyp Entscheidungsfrage mit Frageintention ausgeschlossen. Im Korpus kommt es in sogenannten Vergewisserungsfragen (53), Aufforderungen (54) und Aussagen (55) vor:

(53) aber so ein Dreierteil hast Du doch genommen, ja?

(54) dann nimm doch mal als erstes

(55) das war doch richtig

(56) gibt das Bedeutungsschema für den Aussagesatz (55) an.

(56) Bedeutungsschema für die Modalpartikel doch:

pragmatischer Prätext: im Raum steht: ob das richtig war.

relevante Situation: ich denke: das war richtig

$\rightarrow$ Äußerung: $\quad$ das war doch richtig.

Für die Vergewisserungsfrage (53) kann das gleiche Schema angewandt werden.

(57) Bedeutungsschema für die Modalpartikel doch:

pragmatischer Prätext: $\quad$ im Raum steht: ob Du das Dreierteil genommen hast.

relevante Situation: $\quad$ ich denke: Du hast das Dreierteil genommen

$\rightarrow$ Äußerung: $\quad$ aber so ein Dreierteil hast Du doch genommen, ja?

Die Sprechaktfunktion der "Vergewisserungsfrage" wird im Schema (57) nicht expliziert (für das Bedeutungsschema des verständnisabsichernden ja s. 4.4.). Das Bedeutungsschema unterscheidet sich nicht von dem für die Aussage in (55), was auch dadurch gerechtfertigt ist, daß in (53) dem Strukturtyp nach eine Aussage vorliegt. Für Aufforderungen wie in (54) muß im Schema als relevante Situation statt "ich denke: $p$ " "ich will: p" gesetzt werden.

Im folgenden Beispiel wird deutlich, wie ähnlich sich die Bedeutungen der Modalund der Antwortpartikel doch sind, da sie als Paraphrasen füreinander verwendet werden können:

(58) Habe ich doch gesagt, Mädchen. Doch, habe ich gesagt, da habe ich extra aufgepaßt.

Die Bedeutung von Äußerungen mit doch wird allgemein als 'Widerspruch, Gegensatz' bzw. als adversatives Verhältnis beschrieben (vgl. Ickler 1994:401ff., Abraham 1990:131, Doherty 1985:66, 70). Wir hingegen gehen davon aus, daß die adversative Bedeutung von modalem doch erst durch seine Betonung entsteht und daß die Grundbedeutung von doch nur durch das Aufzeigen von Alternativen gekennzeichnet ist. Entsprechend formuliert der in den Schemata gesetzte pragmatische Prätext, auf den sich doch bezieht, eine Alternative, aus der der Sprecher eine Option auswählt. Man könnte von der Indizierung einer konzessiven Relation sprechen: der 
Sprecher legt sich auf eine der beiden Alternativen fest, wobei er die Existenz einer entgegengesetzten Entscheidung anerkennt. Der Sprecher entscheidet sich für seine Äußerung trotz einer entgegenstehenden Alternative.

Diese Auffassung wird von den Korpusdaten insofern gestützt, als in den Instruktionsdialogen doch sehr häufig in Selektionsaufforderungen gebraucht wird, die dann meist durch Diskurspartikeln, die die Zustimmung des Hörers absichern, begleitet werden (Beispiel (54)).

In der Verwendung von doch als Antwortpartikel, wie in (29), ist das adversative Element dominant.

(29) $\mathrm{K}$ : das hält gar nicht

I: doch, du mußt die andere weiter, äh

Dies rechtfertigt es jedoch nicht, Adversativität als Grundbedeutung von doch anzusetzen. Zum einen ist die Antwortpartikel immer betont, was unsere These bestätigt, daß die adversative Komponente erst suprasegmental entsteht und nicht zur Kernbedeutung der Partikel selbst gehört. Zum zweiten wird das adversative Element der Antwortpartikel dadurch verstärkt, daß die vom Sprecher abgelehnte Alternative, die ja sonst nicht versprachlicht, sondern im pragmatischen Prätext impliziert ist, in diesem Fall vom Partner explizit formuliert wird. Wie die betonte Modalpartikel kennzeichnet die Antwortpartikel die Auswahl der unwahrscheinlicheren Alternative. Die adversative Bedeutung der Antwortpartikel ergibt sich also aus dem intonatorischen und versprachlichten Kontext. Dagegen ist die Grundbedeutung der Partikel doch die Indizierung einer Auswahl aus zwei Alternativen.

\subsection{Ja}

$J a$ tritt als Modalpartikel (fast) nur in Aussagesätzen auf, d.h. es legt den Sprecher auf eine "assertive Haltung" fest (Doherty 1985:78). Mit ja wird die Übereinstimmung des Sprechers mit dem vorausgesetzten mentalen Zustand des Hörers ausgedrückt. Dabei bezieht sich modales ja im Gegensatz zu modalem auch immer nur auf mentale Zustände, nicht auf Äußerungskontexte etc. Es besteht ebenfalls eine Ähnlichkeit zu doch, mit dem Unterschied, daß letzteres einen Kontrast zum Prätext konstatiert, ja dagegen eine Übereinstimmung darstellt (vgl. Doherty 1985:78).

Die Modalpartikel ja, die entweder eine gemeinsame Wahrnehmungsebene, wie in Beispiel (59), oder gleiche Vorannahmen, wie in Beispiel (60) voraussetzt, bezieht sich auf die Annahmen des Hörers (oder einer anderen Person):

(59) Oh dann mußt Du es ja nochmal abmachen

(60) es soll ja auch fliegen

Die Bedeutung des Beispiels (60) läßt sich folgendermaßen auflösen:

(61) Bedeutungsschema für die Modalpartikel ja:

Pragmatischer Prätext: $\quad$ im Raum steht: jemand denkt, daß es auch fliegen soll. 
relevante Situation:

$\rightarrow$ Äußerung: ich denke: es soll auch fliegen.

es soll ja auch fliegen.

Die Bedeutung von modalem $j a$ ist somit paraphrasierbar als 'Indizierung einer Übereinstimmung zwischen den Annahmen des Hörers oder einer anderen Person und des Sprechers'.

Die verschiedenen Funktionen der Partikel ja liegen somit zum einen im modalen Bereich, zum anderen wird $j a$ in den Dialogen als Diskurspartikel verwendet. Hier ist die relationale Grundstruktur am stärksten ausdifferenziert, indem verschiedene kommunikative Ebenen den pragmatischen Kontext und die relevante Situation bilden können. In 3.2. wurde schon gezeigt, wie sich Diskurspartikeln auf den Äußerungskontext, die thematische Struktur und die Verständnisebene beziehen können. So kann auch $j a$ dazu gebraucht werden, ein neues Thema einzuleiten (das Beispiel für diese kommunikative Ebene in 3.2. war aber):

(62) ja ganz genau und jetzt nimmst $D u<$ pause $>$ ja jetzt wird es ein bißchen schwierig

Die äußerst polyfunktionale Diskurspartikel $j a$ kann sich allerdings auf mindestens vier weitere Ebenen beziehen: den Dialogablauf, die Handlungssituation, die Beziehung zwischen den Kommunikationspartnern sowie die Redeformulierungsebene.

Die Ebene des Dialogablaufs betreffend finden sich in den Instruktionsdialogen z.B. Signale der Zustimmung, die sich auf die Gesprächsorganisation beziehen. So geht es in den sogenannten feedback-Signalen nicht um die Zustimmung auf der propositionalen Ebene, sondern um eine Unterstützung im Gesprächsablauf. Den pragmatischen Text konstituiert hierbei der lokutive Akt, d.h. die Äußerungshandlung selbst, die relevante Situation wird konstituiert durch die Wahrnehmung und das Verstehen der Äußerung. Ein Beispiel ist (63), in dem positive Rückmeldung gegeben wird. In dieser Äußerung fungiert $j a$ als Signal der Aufmerksamkeit, des Verständnisses und des Kontakts, also als feedback-Signal, ohne direkte propositionale Zustimmung auszudrücken.

(63) I: Ah ja das das ähm ja das sind die Lager nachher dafür aber ähm

$\mathrm{K}: \underline{J a}$

(64) Bedeutungsschema für $j a$ als feedback-Signal pragmatischer Kontext:

Du hast etwas gesagt

relevante Situation:

$\rightarrow$ Äußerung ich habe gehört, was Du gesagt hast ja

In den hier untersuchten Instruktionsdialogen findet sich zudem eine Verwendung von $j a$, die sich auf die außersprachliche Situation bezieht:

(65) K: Mhm <pause 4 sec $>$ huch $<$ pause 7 sec $>$ ja

Um diese Verwendungsweise zu verstehen, muß man die Situation kennen, in der diese Diskurspartikel geäußert wird: Der Instrukteur gibt eine Bauschrittanleitung an den Konstrukteur, der zunächst die Aufnahme der Instruktion mithilfe von $\mathrm{mhm}$ bestätigt, dann diese Instruktion ausführt und schließlich die Komplettierung des 
jeweiligen Bauschritts mit ja signalisiert. Hier bezieht sich ja also auf die Handlungsebene. Die Übereinstimmung hinsichtlich der Handlungssituation läßt sich in dem relationalen Schema folgendermaßen darstellen:

(66) Bedeutungsschema für $j a$ als Signal einer abgeschlossenen Handlung

$\begin{array}{ll}\text { pragmatischer Kontext: } & \text { Du möchtest, daß ich etwas tue } \\ \text { relevante Situation: } & \text { ich habe es getan } \\ ->\text { Äußerung } & j a\end{array}$

In dem folgenden Austausch bezieht sich die Diskurspartikel ja auf die Beziehungsebene zwischen den Kommunikationspartnern. Als Redeübernahmesignal bestätigt ja das gemeinsame Thema und signalisiert eine grundsätzlich harmonische persönliche Beziehung zwischen den Gesprächspartnern. Diese Interpretation wird dadurch unterstützt, daß ja vor allem in dieser Verwendungsweise gefunden wird, wenn durch den propositionalen Gehalt der Äußerung die Beziehung zwischen den Gesprächspartnern gefährdet ist, wie z.B. in dem folgenden Austausch, in dem der Konstrukteur erklärt, einen Fehler gemacht zu haben:

(67) I: und dann müßtest du noch den mit fünf übrighaben

$\mathrm{K}$ : ja genau ja ich habe eben

I: ja macht <par beg > ja nix wir haben es ja gemerkt <laut lacht $><$ par end $>$

$\mathrm{K}:<$ par beg> einfach den noch < par end > rausgegriffen <laut lacht> den ich mir nicht zur Seite gelegt hatte

I: ja wir haben es ja gemerkt

In dieser Verwendungsweise mildert $j a$ daher potentiell face-threatening acts (FTAs) (Brown \& Levinson 1987) ab. Hier ist die Äußerung, die eingeleitet wird, die Ursache für einen pragmatischen Text, der die Übereinstimmung der Sprecher in Frage stellt. Diese Relation läßt sich folgendermaßen darstellen:

(68) Bedeutungsschema für ja in FTAs

pragmatischer Kontext:

Du hast gesagt: weglegen \& im Raum steht: ich denke nicht wie Du

relevante Situation: ich denke auch: weglegen \& ich habe eben einfach...

$\rightarrow$ Äußerung ja ich habe eben einfach...

Es ist klar, daß der hier ausgewählte pragmatische Text mit dem Rekurs auf grundsätzliche Arbeitsstrategien (hier: die einzubauenden Teile vorher herauszulegen) nur stellvertretend stehen kann für eine Menge an möglichen Aspekten der Übereinstimmung, deren Mitteilung den Sprechern ermöglichen kann, ihre "Gesichter" zu wahren. Dazu gehört beispielsweise das gemeinsame Ziel, das Flugzeug zusammenzubauen, genauso wie die Beziehungsebene zwischen den Sprechern oder die ebenfalls vorhandenen Signale bezüglich der Übernahme desselben Themas. 
Des weiteren kann sich ja auf die Formulierungstätigkeit beziehen, zum Beispiel, um Zeit zur Sprachplanung zu gewinnen:

(69) <pause> ja ähm

Diese Verwendung ist für ja recht selten. Meist wird in dieser Funktion einer der Häsitationsmarker benutzt. Häufiger fungiert ja als Reparaturmarker (Levelt 1983), wobei ja immer vorwärts gerichtet ist, d.h. nicht signalisiert, daß Probleme in der Formulierung auftauchen, sondern daß diese jetzt behoben sind:

(70) < pause > ähm diese <pause > ja diese Rillen

$J a$ signalisiert hier also, daß der Sprecher jetzt weiß, wie er weiterreden kann, daß die Sprachplanung als erfolgreich abgeschlossen betrachtet werden kann. Ja als Reparaturmarker signalisiert eine Übereinstimmung der erforderlichen Sprachplanungsaktivität mit dem tatsächlichen "ich weiß jetzt, was ich sagen will".

Insgesamt ergibt sich somit für die verschiedenen Verwendungsweisen von ja eine relationale Basissemantik, die für die Modal- und die Diskurspartikel ja übereinstimmt, und die in NSM (Wierzbicka 1986) als "ich glaube, wir denken dasselbe" ausgedriickt werden kann. Die Polyfunktionalität von ja entsteht durch die Bezugnahme der relationalen Bedeutungsstruktur auf verschiedene kommunikative Ebenen wie die Annahmen des Hörers für die Modalpartikel und die propositionale Ebene, bzw. den Äußerungskontext, die Redeformulierungsebene, die Handlungssituation, die Beziehung zwischen den Kommunikationspartnern, die Dialogstruktur, die thematische Struktur und die Verständigungsebene für die Diskurspartikel.

\section{Ausblick: Partikeln und Textsorten}

Im Vorhergehenden wurde gezeigt, daß Modal- und Diskurspartikeln eine einheitliche relationale Basissemantik aufweisen. Sie erfüllen verschiedene verknüpfende und verweisende Funktionen, die ihre Einordnung als grammatische Elemente rechtfertigen. Die Polyfunktionalität der vier untersuchten Lexeme resultiert vor allem aus den verschiedenen Bezugselementen, auf die sich die verweisende Komponente der Partikeln richtet.

Abschließend sollen noch einige Anmerkungen zur Textsortenspezifik der verschiedenen Funktionen der Partikeln angefügt werden. So sind z.B. in den untersuchten Instruktionsdialogen aufgrund der gemeinsam zu lösenden Aufgabe die Aspekte der Kooperativität und Partnerbezogenheit stark hervorgehoben. Daher könnten für diese Textsorte die oben dargelegten Bedeutungsschemata diesbezüglich spezifiziert werden. In der Beschreibung des pragmatischen Prätextes für modales $j a$, zum Beispiel, könnte die Position des "jemand" in der Formulierung "im Raum steht: jemand denkt, $\mathrm{p}$ " durchweg durch ein "Du" substituiert werden. 
Ein anderes Beispiel für den Einfluß der Textsorte auf die Verwendung der Partikeln ist die Tatsache, daß modales auch nicht in den Aufforderungen des Korpus gefunden werden konnte. In der Literatur wird diese Verwendungsweise in Sätzen wie

(71) Sei auch brav

zitiert. In den auf Kooperativität ausgerichteten Dialogen des untersuchten Korpus dagegen ist eine solche Verwendung nicht auffindbar, da mit dieser Verwendung eine hierarchische Beziehung zwischen den Sprechern verbunden ist.

Textsortenspezifische Untersuchungen reduzieren zum einen die Komplexität der zu bewältigenden Beschreibungsaufgabe, indem z.B. bestimmte Kommunikationsziele vorausgesetzt werden, die die Interpretationen der Partikeln beeinflussen können. Zum anderen läßt sich umgekehrt durch die Funktionen der Modal- und Diskurspartikeln auf Textsorten zurückschließen: Da sich Diskurspartikeln auf Aspekte des Kommunikationssystems beziehen, ergibt sich eine Eingrenzung ihrer Verwendung auf situierte gesprochene Sprache. In dem Maße, in dem sich Modalpartikeln auf vorausgesetzte Annahmen des Hörers beziehen, sind auch sie an Textsorten gebunden, die in eine Sprecher-Hörer-Situation eingebunden sind.

\section{Zusammenfassung}

Anhand von Äußerungen aus einem Korpus situierter gesprochener Sprache wird für die Partikeln gezeigt, daß ihren modalen und diskursiven Funktionen eine gemeinsame relationale Basissemantik zugrunde liegt, die zwei Aspekte umfaßt: Zum einen eine Verweisstruktur, zum anderen eine lexemspezifische Bedeutung, die die Art der Relation spezifiziert (z.B. als adversative, iterative etc. Relation), die zwischen dem Bezugselement und der partikelhaltigen Äußerung besteht. Diesen Verweischarakter teilen die Partikeln mit grammatischen Morphemen, wie z.B. Tempusflexiven und Proformen, weshalb für die Partikeln ebenfalls ein grammatischer Status angenommen werden kann. Die Polyfunktionalität der vier untersuchten Lexeme als Adverbien, Konjunktionen, Modal- und Diskurspartikeln resultiert aus der Verschiedenheit der Bezugselemente, auf die ihre relationale Basissemantik gerichtet ist. Bei Modalpartikeln ist das Bezugselement ein pragmatischer Prätext, bei Diskurspartikeln sind die Bezugselemente durch verschiedene Aspekte des Kommunikationssystems gegeben.

\section{Literatur}

Abraham, Werner (1990): Zur heterogenen Entfaltung der Modalpartikel im Ahd. und Mhd. In: Anne Betten (Hg.). Neuere Forschungen zur historischen Syntax des Deutschen: Referate der Internationalen Fachkonferenz Eichstätt 1989. Tübingen: Niemeyer. 124-138. (RGL 103). 
Abraham, Werner (1991): Dicourse particles in German: How does their illocutive force come about? In: ders. (ed.). Discourse particles. Descriptive and theoretical investigations on the logical, syntactic and pragmatic properties of discourse particles in German. Amsterdam, Philadelphia: Benjamins. 203-252. (Pragmatics and beyond, new series, 12).

Brown, Penelope \& Levinson, Stephen (1987): Politeness: Some Universals in Language Usage. Cambridge: Cambridge University Press.

Bühler, Karl (1982 [1934]): Sprachtheorie. Die Darstellungsfunktion der Sprache. Mit einem Geleitwort von Friedrich Kainz. Stuttgart, New York: Fischer.

Burkhardt, Armin (1994): Abtönungspartikeln im Deutschen: Bedeutung und Genese. ZGL, 22, 129-151

Diewald, Gabriele (1991): Deixis und Textsorten im Deutschen. Tübingen: Niemeyer. (RGL 118).

Doherty, Monika (1985): Epistemische Bedeutung. Berlin: Akademie-Verlag. (studia grammatica 23).

Eisenberg, Peter (1994): Grundriß der deutschen Grammatik. 3. überarb. Aufl. Stuttgart: Metzler.

Fischer, Kerstin (1996): A Construction-based Approach to the Lexicalization of Interjections. In: Gellerstam, M., Järborg, J., Malmgren, S., Nörén, K., Rogström, L. and Rojder Papmehl, C. (eds.): Euralex '96: Proceedings. University of Göteborg.

Fischer, Kerstin (forthcoming): Review of: René Dirven \& Johan Vanparys: Current Approaches to the Lexicon. Peter Lang, 1995. Journal of Lexicology.

Fischer, Kerstin \& Johanntokrax, Michaela (1995): Ein linguistisches Merkmalsmodell für die Lexikalisierung von diskurssteuernden Partikeln. SFB 360 "Situierte künstliche Kommunikatoren", Report 18.

Gelhaus, Hermann (1995): Die Wortarten. In: Duden: Grammatik der deutschen Gegenwartssprache. 5., völlig neu bearbeitete und erweiterte Auflage. $\mathrm{Hg}$. u. bearbeitet von Günter Drosdowski [u. a.]. Mannheim usw.: Dudenverlag. 85-398. (Duden 4).

Gülich, Elisabeth (1970): Macrosyntax der Gliederungssignale im gesprochenen Französisch. München: Fink.

Helbig, Gerhard und Joachim Buscha (1986): Deutsche Grammatik: Ein Handbuch für den Ausländerunterricht. Leipzig: Enzyklopädie.

Hentschel, Elke (1986): Funktion und Geschichte deutscher Partikeln: "Ja", "doch", "halt" und "eben". Tübingen: Niemeyer. (RGL 63).

Ickler, Theodor (1994): Zur Bedeutung der sogenannten "Modalpartikeln". Sprachwissenschaft 19, 374-404.

Levelt, Willem J.M. (1983): Monitoring and Self-Repair in Speech. Cognition 14, 41-104.

Östman, Jan-Ola (1981): You Know: A Discourse-Functional Approach. Amsterdam: John Benjamins. (Pragmatics \& Beyond II:7).

Petrič, Teodor (1995): Indexikalische Leistungen der Modalpartikeln und ihre natürlichkeitstheoretische Bewertung. Linguistica 35, 245-259. 
Sagerer, G. \& Eikmeyer, H.J. \& Rickheit, G. (1994): Wir bauen jetzt ein Flugzeug. Konstruieren im Dialog. Arbeitsmaterialien. Tech. Report, SFB 360 "Situierte künstliche Kommunikatoren, University of Bielefeld.

Schiffrin, Deborah (1987): Discourse markers. Cambridge: Cambridge University Press.

Schourup, Lawrence C. (1983): Common Discourse Particles in English Conversation. Working papers in linguistics, 28, The Ohio State University, Department of Linguistics.

Weydt, Harald (1969): Abtönungspartikel. Die deutschen Modalwörter und ihre französischen Entsprechungen. Bad Homburg, Berlin, Zürich: Gehlen

Weydt, Harald (Hg.) (1983). Partikeln und Interaktion. Tübingen: Niemeyer.

Weydt, Harald (Hg.) (1989): Sprechen mit Partikeln. Berlin, New York: de Gruyter.

Wierzbicka, Anna (1986): A semantic metalanguage for the description and comparison of illocutionary meanings. Journal of Pragmatics 10, 67-107.

Wierzbicka, Anna (1991): Cross-cultural pragmatics. The semantics of human interaction. Berlin: Mouton, De Gruyter.

Wierzbicka, Anna (1992): The semantics of interjections. Journal of Pragmatics 18, 159-192.

Wilkins, David (1992): Interjections as deictics. Journal of Pragmatics 18, 245-271. Willkop, Eva-Maria (1988): Gliederungssignale im Dialog. München: Iudicium.

Povzetek

\section{O DISKURZIVNI IN MODALNI FUNKCIJI ČLENKOV $A B E R, A U C H, D O C H$ IN $J A$ V NAVODILNIH DIALOGIH}

Na podlagi analize iz korpusa situacijsko vezanega govorjenega jezika avtorici opozorita na dejstvo, da modalne in diskurzivne funkcije členkov temeljijo na skupni relacijski pomenski osnovi, ki obsega dva vidika: po eni strani gre za strukturo navezovanja, po drugi pa za leksemsko specifični pomen, ki opredeljuje vrsto relacije (npr. protivno, ponavljalno idr.), ki obstaja med nanašalnico in povedjo, ki vsebuje členek. Ta nanašalni značaj si delijo členki s slovničnimi morfemi kot npr. temporalni morfemi in zaimki, zaradi česar lahko predpostavljamo, da imajo tudi členki svoj slovnični status. Polifunkcionalnost štirih raziskanih leksemov kot prislovov, veznikov, modalnih in diskurzivnih členkov izhaja iz različnosti nanašalnic, na katere je usmerjena njihova osnovna relacijska semantika. Pri modalnih členkih je nanašalnica predhodno pragmatično besedilo, pri diskurzivnih členkih pa temeljijo nanašalnice na različnih vidikih sistema komunikacije. 